\title{
For Better or Worse: The Effect of Levodopa on Speech in Parkinson's Disease
}

\author{
Aileen K. Ho $\mathrm{PhD},{ }^{1} *$ John L. Bradshaw $\mathrm{DSc},{ }^{2}$ and Robert Iansek FRACP ${ }^{3}$ \\ ${ }^{1}$ Department of Psychology, School of Psychology and Clinical Language Sciences, University of Reading, Reading RG6 6AL, \\ United Kingdom \\ ${ }^{2}$ School of Psychology, Psychiatry and Psychological Medicine, Monash University, Clayton 3168, Victoria, Australia \\ ${ }^{3}$ Geriatric Neurology Research Unit, Kingston Centre, Cheltenham 3192, Victoria, Australia
}

\begin{abstract}
While the beneficial effect of levodopa on traditional motor control tasks have been well documented over the decades, its effect on speech motor control has rarely been objectively examined and the existing literature remains inconclusive. To examine the effect of levodopa on speech in patients with Parkinson's disease, it was hypothesized that levodopa would improve preparatory motor set related activity and alleviate hypophonia. Patients fasted and abstained from levodopa overnight. Motor examination and speech testing was performed the following day, pre-levodopa during their "off " state, then at hourly intervals postmedication to obtain the best "on" state. All speech stimuli
\end{abstract}

showed a consistent tendency for increased loudness and faster rate during the "on "state, but this was accompanied by a greater extent of intensity decay. Pitch and articulation remained unchanged. Levodopa effectively upscaled the overall gain setting of vocal amplitude and tempo, similar to its well-known effect on limb movement. However, unlike limb movement, this effect on the final acoustic product of speech may or may not be advantageous, depending on the existing speech profile of individual patients. (C) 2007 Movement Disorder Society

Key words: Parkinson's disease; levodopa; speech; intensity; volume or intensity decay; duration
The advent of levodopa (L-dopa) therapy in the late 1960s heralded a new era in the treatment of Parkinson's disease (PD), and this is still the most widespread form of intervention today. Nevertheless, although the beneficial effect of L-dopa on limb motor control tasks has been well-documented over the decades, the same cannot be said for speech motor control. Cerebral activation studies, however, have shown the importance of the basal ganglia (BG) and cerebellum for both the execution of $\operatorname{limb}^{1}$ and speech movements, 2,3 in PD.

While speech is important in everyday functioning, and impacts upon quality of life, there is a paucity of speech pharmacokinetic research in PD. ${ }^{4,5}$ Furthermore,

*Correspondence to: Dr. Aileen Ho, Department of Psychology, School of Psychology and Clinical Language Sciences, University of Reading, Reading RG6 6AL, United Kingdom.

E-mail: aileenkho@netscape.net

Received 21 November 2006; Revised 9 November 2007; Accepted 11 November 2007

Published online in Wiley InterScience (www.interscience.wiley. com). DOI: $10.1002 / \mathrm{mds} .21899$ the existing body of research reveal mixed findings. ${ }^{6,7} \mathrm{~A}$ key point that emerges from the literature is the high level of variability between patients, and across measures, for example, kinematic studies have found varied performance between patients, individual articulators, and different measures. ${ }^{8-12}$ While these kinematic and electromyography studies ${ }^{13-15}$ attempt to tap into the neurophysiology of the speech articulators, they suffer from several disadvantages, which may account in part for the varied findings. First, these measures are intrusive and contact with the articulators may well interfere with and alter the nature of the speech task by drawing attention to it. ${ }^{16,17}$ Second, the specific contribution of individual articulators may be too localized to be meaningful since there are multiple degrees of freedom in coordination of the complex speech apparatus and the same speech output can be achieved in a variety of ways via motor equivalence. ${ }^{8,18}$ Third, impairment of peripheral speech articulators may be attributed to neuromuscular denervation or muscular atrophy rather than higher order dysfunction. ${ }^{19,20}$ Therefore, there is a considerable theo- 
retical and empirical research gap in impaired speech motor control in PD, and the paucity of speech pharmacokinetic research has made it difficult to ascertain whether our current understanding of general limb motor control extends to speech motor control.

One approach has been to examine the acoustic endproduct of the speech motor apparatus since this is objective and non-intrusive. The rationale behind this is that speech articulation is essentially a series of skilled motor gestures that require upstream central coordination of fundamental higher-order processes for skilled motor control which transcends effector systems. These preparatory motor processes such as motor planning and programming, are critically mediated by cerebral networks for speech production, including the frontostriatal motor circuit. Thus, dysfunction of the BG such as in PD may result in characteristically similar motor preparation and execution impairments in both systems, especially in the sequencing aspect common to both articulation and limb movement.

Movement preparation is characterized by a sustained premovement SMA neural discharge, and is thought to contribute to cortical motor set, which allows the forthcoming movement to be normally executed. ${ }^{21}$ Motor set thus refers to a state of readiness to make a movement. ${ }^{22}$ In PD, hypokinesia resulting in short-stepped shuffling gait $^{23}$ and miniaturized handwriting ${ }^{24}$ may be due to insufficient motor set. Our previous work suggests a corresponding analogue in reduced speech intensity. We found that while PD patients were able to adjust or scale the loudness of their voice and produce different levels of intensity according to task demands, they were nevertheless always quieter than healthy participants by a constant amount at every intensity level. ${ }^{25,26} \mathrm{We}$ and others $^{27}$ have also shown that PD patients can indeed generate sufficiently loud volume under certain circumstances (e.g. by using explicit instructions which direct the focus of attention to the task), illustrating that like limb movement, the motor program is essentially intact but is insufficiently activated under normal circumstances.

During the execution phase of movement, tonic SMA and phasic discharges from the globus pallidus (GP) interact to effect the smooth execution of automatic skilled movement via the BG-thalamocortical motor circuit. The GP is critically involved in the internal release of each submovement within a motor sequence. ${ }^{28}$ Once the first submovement is executed, the GP would produce a phasic burst of activity to trigger the crisp termination of SMA set-related preparatory activity and the release of the next submovement, while permitting setrelated activity for the following submovement to com- mence. Hence, the entire skilled movement sequence can be automatically run to completion. ${ }^{29}$ In PD, faulty GP phasic output to the SMA may lead to abnormal preparatory activity resulting in submovements that are slow and reduced in amplitude; these impairments may compound as the sequence progresses, resulting in motor instability. Therefore, the amplitude of submovements can be further diminished as the execution of the sequence progresses. This sequencing effect (also known as "motor instability") refers to the inability to maintain the preset amplitude for each submovement ${ }^{30,31}$ and has been shown in the control of the upper and lower limbs. Progressive decreases in movement amplitude over the duration of a motor sequence in PD has been demonstrated in a repetitive finger movement task ${ }^{32,33}$ micrographic handwriting, ${ }^{24}$ festination in walking, ${ }^{23}$ and fading speech. ${ }^{34}$

Our work provides behavioral evidence suggesting underlying similarities across speech and limb motor systems of PD patients, and specifically, that hypophonia (reduced speech volume or intensity) may be the speech analogue of limb hypokinesia (reduced movement extent) and bradykinesia (slowed movement). This appears to be the key feature of Parkinsonian hypokinetic dysarthria ${ }^{35-37}$ and the best target for focused effort in speech therapy, ${ }^{38,39}$ or more recently, combined speech and physical therapy. ${ }^{40}$ The role of the BG in the regulation of force or power ${ }^{41}$ may indeed underlie the spatial (articulator and limb undershoot) and temporal (slowed speech and limb movement) poverty of skilled movement across effector systems. Limb control studies has shown that L-dopa can normalize preparatory motor set and increase the average stride length of PD patients, nevertheless, motor instability (or the sequence effect) was not improved. ${ }^{42}$ In speech, while perceptual studies have documented higher intelligibility ratings when on L-dopa, which might (in part) be due to an overall increment in speech volume, ${ }^{43,44}$ this has rarely been objectively examined. Furthermore, there are no published reports examining the effect of L-dopa motor instability or speech fading. Therefore, in this study we examine the effect of L-dopa on these key dimensions of speech using acoustic analysis, and specifically predict that similar to gait, ${ }^{42}$ L-dopa would only improve overall motor set (resulting in increased overall speech intensity), and not progressive motor instability.

\section{PATIENTS AND METHODS}

\section{Participants and Procedure}

The participants were nine mild to moderate nondemented idiopathic PD patients diagnosed according to 
TABLE 1. Clinical data for patients with Parkinson's disease

\begin{tabular}{|c|c|c|c|c|c|c|c|}
\hline Patient & Age (yr) & Sex & STMS* & $\begin{array}{l}\text { Disease duration } \\
\text { (yr) }\end{array}$ & $\begin{array}{l}\text { UPDRS }^{* * *} \text { "Off" } \\
\text { state }\end{array}$ & $\begin{array}{l}\text { UPDRS speech } \\
\text { item** "Off" state }\end{array}$ & $\begin{array}{l}\text { Levodopa equivalent } \\
\text { test dose }(\mathrm{mg})\end{array}$ \\
\hline 1 & 56 & $\mathrm{M}$ & 36 & 10 & 9 & 1 & 200 \\
\hline 2 & 71 & M & 32 & 5 & 19 & 1 & 125 \\
\hline 3 & 70 & $\mathrm{M}$ & 33 & 10 & 18 & 1 & 250 \\
\hline 4 & 67 & $\mathrm{M}$ & 29 & 15 & 15 & 1 & 200 \\
\hline 5 & 69 & $\mathrm{M}$ & 29 & 18 & 22 & 2 & 200 \\
\hline 6 & 84 & M & 29 & 3 & 11 & 1 & 150 \\
\hline 7 & 72 & M & 34 & 5 & 16 & 1 & 250 \\
\hline 8 & 52 & $\mathrm{~F}$ & 36 & 6 & 20 & 1 & 440 \\
\hline 9 & 80 & $\mathrm{M}$ & 25 & 3 & 21 & 1 & 150 \\
\hline
\end{tabular}

* Short test of mental status. ${ }^{46}$

**Unified Parkinson's disease rating scale. ${ }^{47}$

the UK brain bank criteria, ${ }^{45}$ who were on a stable medication regime (see Table 1 for clinical details). Patients in a movement disorder clinic were examined by an experienced neurologist (RI) with extensive specialist experience in PD, and consecutive patients with predominantly hypophonic speech presentation were recruited to the study. Anti-Parkinson medication was stopped for least 12 hours overnight to induce a practically-defined "off " state according to the well-recognized CAPIT protocol. ${ }^{48}$ The Unified Parkinson's Disease Rating Scale (UPDRS) ${ }^{47}$ motor examination and speech testing was conducted before the first dose of L-dopa medication on the following morning, and thereafter at hourly intervals up to 3 hours post-medication to obtain the best "on " state. The UPDRS speech item score on the UPDRS at predose was $\geq 1$ for all participants. As per the CAPIT protocol, these patients were also fasted overnight and up to the end of the experiment, although water was permitted.
We mapped drug response using UPDRS motor scores. On the basis of individual dose-response curves, we ascertained when individual patients were "on " or "off" in the medication cycle. "On" was defined as either a $25 \%$ or greater improvement from baseline UPDRS score, or between $15 \%$ and $25 \%$ improvement from baseline but with no less that $20 \%$ drop from best postmedication score. These two criteria captured the major changes in their motor profile in response to L-dopa (see Fig. 1). There was indeed a statistically significant group difference $(\mathrm{F}(1,8)=29.68, P<0.05)$ between "off " $(17.03 \pm 1.73)$ "on " $(9.19 \pm 1.57)$ scores.

\section{Speech Stimuli}

Speech data were likewise individually classified as occurring during the "on" or "off" phase based on the UPDRS criteria. Speech stimuli consisted of (1) sustained vowel phonation, (2) counting, and (3) conversation. In the first two conditions, participants were in-

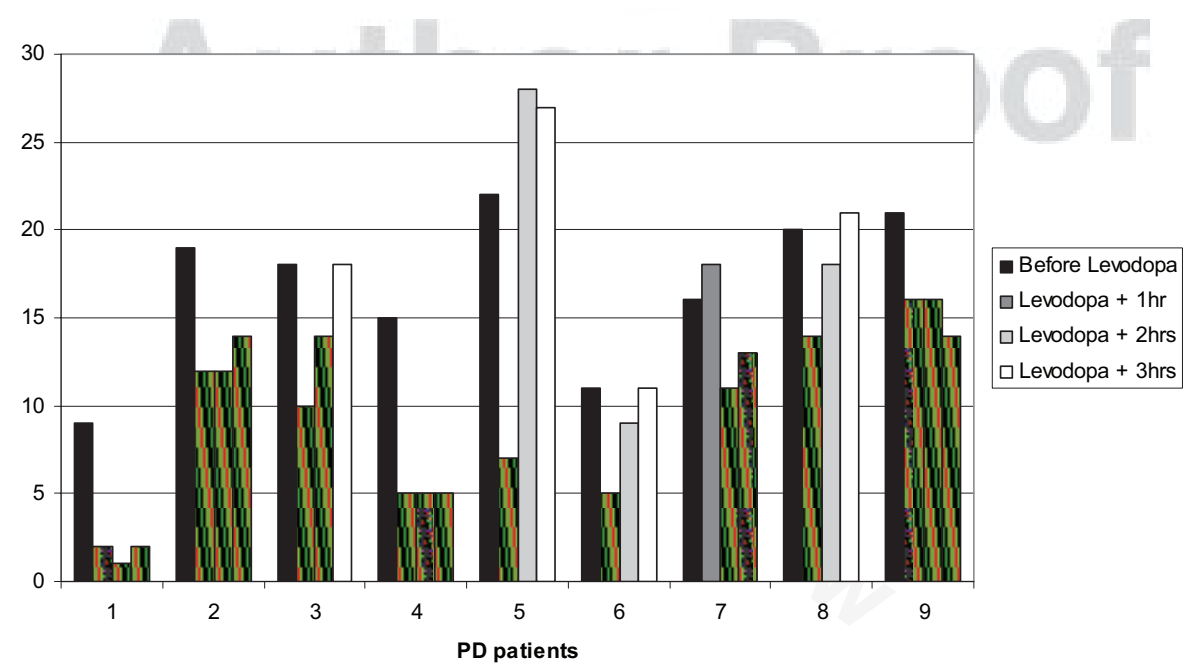

FIG. 1. UPDRS motor scores across the L-dopa cycle. Striped bars indicate "on " phase. 
structed to take a deep breath before starting. Conversation was elicited by asking participants to talk about familiar topics, such as their house or garden. Participants were familiarized with all three types of speech stimuli on a separate occasion prior to testing. All speech conditions were performed under three volume instructions; as quietly as possible without whispering, as loudly as possible without shouting, and without volume instruction in order to elicit self-selected normal volume. The same experimenter conducted the study and on all occasions the same set of instructions was used to elicit the speech stimuli, thereby the normal volume condition was elicited first, followed by quiet then loud condition. Using a David Clarke head-set, a mouth-to-microphone distance of $8 \mathrm{~cm}$ was kept constant and speech was audio-taped using a Marantz tape recorder (PMD222) with the gain control setting at a fixed position. The data were later digitized (sampling rate $22 \mathrm{kHz}$ ) and analyzed using Multi-Speech (Kay Elemetrics). ${ }^{49}$ For the conversation condition, 30-second samples of free flowing speech were extracted. Pauses, as defined by no vocal output for a duration of 200 millisecond or longer, ${ }^{50}$ were determined from the waveform and raw energy displays of the speech sample, and manually removed.

\section{RESULTS}

\section{Overall Analysis}

Speech measures examined were average (mean) intensity, intensity decay (as indexed by linear regression slopes of intensity over time), and duration (of speech within one breath envelope). A three-way ANOVA with factors of stimuli (sustained phonation, counting, conversation), instruction level (quiet, normal, and loud volume), and medication phase (on, off) was performed on these measures.

For mean intensity, there were main effects of stimuli $(\mathrm{F}(2,16)=15.07, P<0.05)$ and instruction $(\mathrm{F}(2,16)=$ 21.12, $P<0.05)$, which interacted significantly $(\mathrm{F}(4,32)=11.38, P<0.05)$, but critically, did not interact with medication. There was, however, a strong trend for a medication main effect $(\mathrm{F}(1,8)=4.22, P=$ $0.07)$ where the "on " phase $(56.77 \pm 1.65 \mathrm{~dB})$ was louder than "off" $(56.12 \pm 1.54 \mathrm{~dB})$ across all conditions (see Fig. 2).

For intensity decay, there were no significant interactions. There were significant main effects of stimuli $(\mathrm{F}(2,16)=5.19, P<0.05)$ and instruction $(\mathrm{F}(2,16)=$ $4.11, P<0.05)$, and a strong trend for a medication main effect $(\mathrm{F}(1,8)=4.77, P=0.06)$ where intensity decay was greater when "on" $(-0.72 \pm 0.14)$ than "off" $(-0.61 \pm 0.13)$ (see Fig. 2).
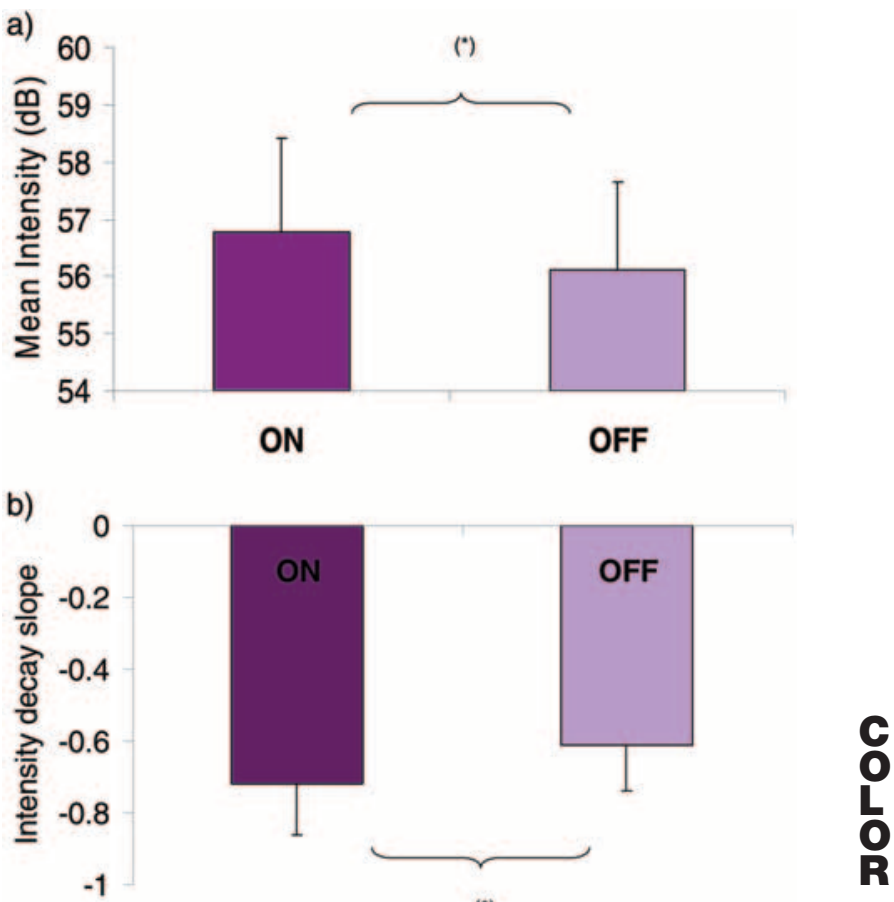

FIG. 2. Mean intensity (a) and intensity decay (b) data for all speech stimuli during patients" "on" and "off" phases. (*), $P<0.10$.

For speech duration, there was only a significant main effect of stimuli $(\mathrm{F}(2,16)=33.45, P<0.05)$, as expected, since conversation breath spans were naturalistic unlike maximal vowel phonation and counting tasks. The absence of a significant main effect of instruction indicated that lung capacity was not a limiting factor since patients were able to speak at all loudness levels for a similar period of time. Notably, there was no significant medication main effect or interactions.

\section{Detailed Subanalysis on Normal Intensity Counting}

Subsequent analyses required carefully controlled speech stimuli which were the same between participants, and were therefore necessarily restricted to counting data. Counting at normal vocal intensity was used because this was performed at the individuals' self-selected speech intensity and would therefore be more natural and representative. Speech measures examined were mean intensity, intensity decay, breath span counting duration, rate (syllables per second), average (mean) fundamental frequency (F0), F0 range, voice onset time (VOT) of the plosive/t/in "two" (i.e. time from articulatory release of/t/to the onset of voicing for the vowel/ $\mathrm{u} /$ ), and intensity during closure (IDC) of the plosive/t/in "two" (i.e. lowest point in the sound pressure level contour during the alveolar stop closure). 
a)

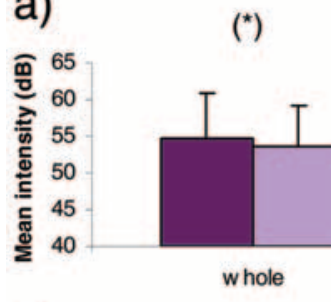

b)

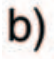

Counting Mean Intensity

NS $\left({ }^{\star}\right)$

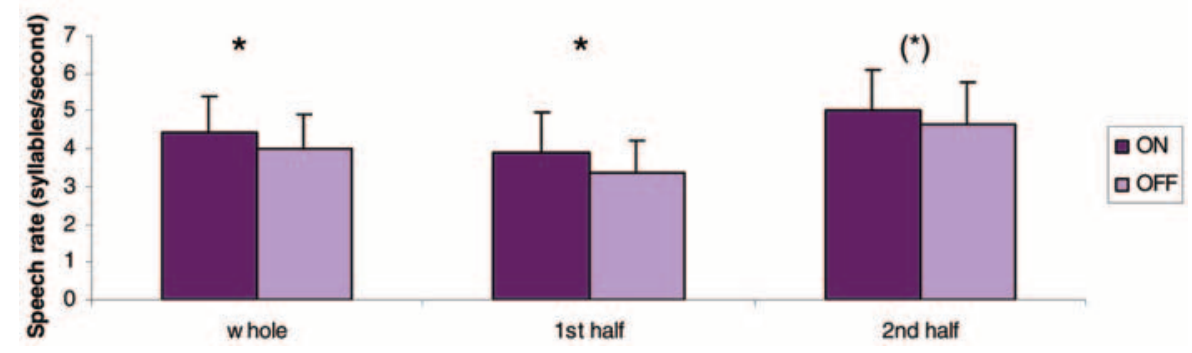

FIG. 3. Mean intensity (a) and rate (b) data for normal intensity counting during patients' "on " and "off" phases. *, $P<0.05 ;(*), P<0.10$; NS, not significant.

Mean intensity for the entire counting sequence when "on " (54.85 $\pm 6.05 \mathrm{~dB})$ was significantly louder $(\mathrm{F}(1,8)=3.42, P<0.05)$ than when "off" (53.62 \pm $5.61 \mathrm{~dB})$, see Figure 3. This was seen as a trend $(\mathrm{F}(1,8)=3.51, P=0.098)$ for the second half of the sequence ("off" = $51.42 \pm 5.55 \mathrm{~dB}$; "on " $=52.60 \pm$ $5.88 \mathrm{~dB}$ ) but not the first, reflecting some decay of intensity over the time course of counting. However, the greater "on" phase intensity decay was slight and did not reach significance when the linear regression slope was analyzed $(\mathrm{F}(1,8)=2.66, P=0.24)$.

Counting rate was significantly $(\mathrm{F}(1,8)=6.17, P<$ $0.05)$ faster "on " (4.41 \pm 1.01 sylls/second) than “off"(4.00 \pm 0.93 sylls/second), see Figure 3. This pattern was significant $(\mathrm{F}(1,8)=5.90, P<0.05)$ for the first half of the sequence, and was borderline significant $(\mathrm{F}(1,8)=5.24, P=0.05)$ in the second half.

There were no significant differences between "on " and "off" phases for counting duration, average F0, F0 range, VOT, and IDC.

Correlations were carried out between factors which showed an effect of medication. The increase in mean intensity from "off" to "on " correlated with increased "on" counting rate for the whole sequence $\left(\mathrm{r}^{2}=0.74\right.$, $P<0.05)$, first half $\left(\mathrm{r}^{2}=0.75, P<0.05\right)$, and second half $\left(\mathrm{r}^{2}=0.64, P=0.06\right)$. Speech rate was also significantly associated with UPDRS performance, such that PD patients who counted more slowly at the start of breath span when "off" were likely to have higher (i.e. poorer) motor scores when "on" $\left(\mathrm{r}^{2}=-0.70, P<0.05\right)$.

\section{DISCUSSION}

This study examined the effect of L-dopa on speech, and more specifically, the notion that there may be parallel effects on the speech and skeletal motor system.

During the "on" phase, improvement in UPDRS motor performance was accompanied by an increase in the overall level of speech intensity. While some studies have found no dopaminergic effect on overall speech intensity, ${ }^{51-54}$ others have found some support for this. ${ }^{55-58}$ The second study reported a single case and the latter study noted that two of seven patients improved, suggesting that the discrepancy in past findings could perhaps be partly due to individual patient characteristics. It is therefore important to recognize the different speech profiles of PD patients, ${ }^{36}$ beyond that which can be captured in the UPDRS speech item. This study selectively examined patients with predominantly hypophonic speech, followed their individual response to L-dopa over the course of the medication cycle, and engaged them in carefully breath-span controlled speech tasks that were sufficiently naturalistic. All or part of these points of difference with studies reporting a null effect would appear to be important, and should therefore be considered in the development of future studies.

The upscaling of motor intent across speech and limb control is consistent with an upregulation of gain programming for the motor set of the entire movement sequence. $31,34,42$ Furthermore, speech rate increased and was significantly correlated with mean intensity increase. 
This is similar to the L-dopa induced upscaling of gain for limb movement speed and extent. ${ }^{58,59}$ The parallel between speech and limb motor control is also supported by the significant inverse correlation between speech rate and UPDRS, whereby patients who counted more slowly at the start of breath span when "off" were likely to have higher poorer motor scores when "on."

The maintenance of speech intensity over successive speech segments over the breath span did not benefit from L-dopa, suggesting that this may be influenced by a separate motor cueing mechanism responsible for submovement regulation. ${ }^{31,34}$ This is the first dose-response study to examine any progressive change in speech intensity within speech sequences completed in a breath span. The lack of dopaminergic benefit is strikingly similar to the absence of drug-effect on altering progressively diminished stride length in festinating gait in a recent study. ${ }^{42}$

These findings above were applicable to all three types of speech stimuli used, i.e. sustained phonation, counting, and conversation. This suggests that dopaminergic medication influences the basic speech production mechanism underpinning the execution of speech stimuli of different levels of complexity. Further work is needed to fully investigate this, as well as the contribution of key structures such as the BG, SMA and also cerebellum which are implicated in motor control and are also affected in PD.

Taken together, while the speech changes reported may be small (see Fox and Ramig, 1997), ${ }^{60}$ they are hypothesis-driven, specific, and consistent. We found that the effect of L-dopa on the primary speech measures of intensity and rate, are compatible with the limb movement literature, and provide a parsimonious account for the varied findings in Parkinsonian speech. Although these initial results are limited by sample size and will clearly need to be rigorously investigated in a largerscale study, they are novel and provide a timely counterpoint to the null effect findings in the literature suggesting that speech is perhaps not entirely impervious to dopaminergic influence. It is conceivable that PD patients with different types of predominant speech manifestation will show different responses to L-dopa medication as a direct result of the unique interaction of the dopaminergic upscaling effect and their specific speech profiles. With this context in mind, fluency problems may improve only if speech is slow enough. Intelligibility may be perceived to improve due to increased overall speech intensity, but if compounded with a "festinating" fluency problem (which may be exaggerated by even greater intensity decay), there may not be a perceptible net improvement. Therefore, the interplay between the direct effects of L-dopa in upscaling speech intensity and rate, and any indirect compensatory changes will ultimately determine the net effect of medication on each patient's existing speech profile.

Acknowledgments: The authors gratefully acknowledge John Cockayne Fellowship Fund, Australia and the assistance of C. Burns in data processing.

\section{REFERENCES}

1. Wu T, Hallett M. A functional MRI study of automatic movements in patients with Parkinson's disease. Brain 2005;128:2250-2259.

2. Liotti M, Ramig LO, Vogel D, et al. Hypophonia in Parkinson's disease: neural correlates of voice treatment revealed by PET. Neurology 2003;60:432-440.

3. Riecker A, Mathiak K, Wildgruber D, et al. fMRI reveals two distinct cerebral networks subserving speech motor control. Neurology 2005;64:700-706.

4. Sanabria J, Ruiz PG, Gutierrez R, et al. The effect of levodopa on vocal function in Parkinson's disease. Clin Neuropharmacol 2001; 24:99-102.

5. Goberman AM, Coelho C. Acoustic analysis of parkinsonian speech. I. Speech characteristics and L-Dopa therapy. NeuroRehabilitation 2002;17:237-246.

6. Schulz GM, Grant MK. Effects of speech therapy and pharmacologic and surgical treatments on voice and speech in Parkinson's disease: a review of the literature. J Commun Disord 2000;33:5988.

7. Pinto S, Ozsancak C, Tripoliti E, Thobois S, Limousin-Dowsey P, Auzou P. Treatments for dysarthria in Parkinson's disease. Lancet Neurol 2004;3:547-556.

8. Forrest K, Weismer G, Turner GS. Kinematic, acoustic, and perceptual analyses of connected speech produced by parkinsonian and normal geriatric adults. J Acoust Soc Am 1989;85:2608-2622.

9. Connor NP, Abbs JH, Cole KJ, Gracco VL. Parkinsonian deficits in serial multiarticulate movements for speech. Brain 1989;112 (Part 4):997-1009.

10. Ackermann H, Grone BF, Hoch G, Schonle PW. Speech freezing in Parkinson's disease: a kinematic analysis of orofacial movements by means of electromagnetic articulography. Folia Phoniatr (Basel) 1993:45:84-89.

11. Forrest K, Weismer G. Dynamic aspects of lower lip movement in parkinsonian and neurologically normal geriatric speakers' production of stress. J Speech Hear Res 1995;38:260-272.

12. Ackermann H, Hertrich I, Daum I, Scharf G, Spieker S. Kinematic analysis of articulatory movements in central motor disorders. Mov Disord 1997;12:1019-1027.

13. Netsell R, Cleeland CS. Modification of lip hypertonia in dysarthria using EMG feedback. J Speech Hear Disord 1973;38:131140.

14. Hunker CJ, Abbs JH, Barlow SM. The relationship between parkinsonian rigidity and hypokinesia in the orofacial system: a quantitative analysis. Neurology 1982;32:749-754.

15. Baker KK, Ramig LO, Luschei ES, Smith ME. Thyroarytenoid muscle activity associated with hypophonia in Parkinson disease and aging. Neurology 1998;51:1592-1598.

16. Ackermann H, Ziegler W. Articulatory deficits in parkinsonian dysarthria: an acoustic analysis. J Neurol Neurosurg Psychiatry 1991;54:1093-1098.

17. Ho AK, Iansek R, Bradshaw JL. The effect of a concurrent task on Parkinsonian speech. J Clin Exp Neuropsychol 2002;24:36-47.

18. Ho AK, Bradshaw JL, Cunnington R, Phillips JG, Iansek R. Sequence heterogeneity in Parkinsonian speech. Brain Lang 1998; 64:122-145.

19. Gath I, Yair E. Analysis of vocal tract parameters in parkinsonian speech. J Acoust Soc Am 1988;84:1628-1634. 
20. Smith ME, Ramig LO, Dromey C, Perez KS, Samandari R. Intensive voice treatment in Parkinson disease: laryngostroboscopic findings. J Voice 1995;9:453-459.

21. Alexander GE, Crutcher MD. Preparation for movement: neural representations of intended direction in three motor areas of the monkey. J Neurophysiol 1990;64:133-150.

22. Evarts EV, Wise SP. Basal gangalia outputs and motor control. Ciba Foundation Symp 1984;107:83-102.

23. Morris ME, Iansek R, Matyas TA, Summers JJ. The pathogenesis of gait hypokinesia in Parkinson's disease. Brain 1994;117 (Part 5):1169-1181

24. Oliveira RM, Gurd JM, Nixon P, Marshall JC, Passingham RE. Micrographia in Parkinson's disease: the effect of providing external cues. J Neurol Neurosurg Psychiatry 1997;63:429-433.

25. Ho AK, Iansek R, Bradshaw JL. Regulation of parkinsonian speech volume: the effect of interlocuter distance. J Neurol Neurosurg Psychiatry 1999;67:199-202.

26. Ho AK, Bradshaw JL, Iansek R, Alfredson R. Speech volume regulation in Parkinson's disease: effects of implicit cues and explicit instructions. Neuropsychologia 1999;37:1453-1460.

27. Ramig LO. How effective is the Lee Silverman voice treatment? Asha 1997;39:34-35.

28. Brotchie P, Iansek R, Horne MK. Motor function of the monkey globus pallidus. I. Neuronal discharge and parameters of movement. Brain 1991;114 (Part 4):1667-1683.

29. Brotchie P, Iansek R, Horne M. A neural network model of neural activity in the monkey globus pallidus. Neurosci Lett 1991;131: 33-36.

30. Martin KE, Phillips JG, Iansek R, Bradshaw JL. Inaccuracy and instability of sequential movements in Parkinson's disease. Exp Brain Res 1994;102:131-140.

31. Iansek R, Bradshaw JL, Phillips JG, Cunnington R, Morris ME. Interaction ofthe basal ganglia and supplementary motor area in the elaboration of movement. Adv Psychol (Amsterdam) 1995;111: 37-60.

32. Konczak J, Ackermann H, Hertrich I, Spieker S, Dichgans J. Control of repetitive lip and finger movements in Parkinson's disease: influence of external timing signals and simultaneous execution on motor performance. Mov Disord 1997;12:665-676.

33. Agostino R, Berardelli A, Curra A, Accornero N, Manfredi M. Clinical impairment of sequential finger movements in Parkinson's disease. Mov Disord 1998;13:418-421.

34. Ho AK, Iansek R, Bradshaw JL. Motor instability in parkinsonian speech intensity. Neuropsychiatry Neuropsychol Behav Neurol 2001;14:109-116.

35. Logemann JA, Fisher HB, Boshes B, Blonsky ER. Frequency and cooccurrence of vocal tract dysfunctions in the speech of a large sample of Parkinson patients. J Speech Hear Disord 1978;43:4757.

36. Ho AK, Iansek R, Marigliani C, Bradshaw JL, Gates S. Speech impairment in a large sample of patients with Parkinson's disease. Behav Neurol 1998;11:131-137.

37. Kent RD, Kim YJ. Toward an acoustic typology of motor speech disorders. Clin Linguist Phon 2003;17:427-445.

38. Ramig LO, Sapir S, Fox C, Countryman S. Changes in vocal loudness following intensive voice treatment (LSVT) in individuals with Parkinson's disease: a comparison with untreated patients and normal age-matched controls. Mov Disord 2001;16:79-83.

39. Trail M, Fox C, Ramig LO, Sapir S, Howard J, Lai EC. Speech treatment for Parkinson's disease. NeuroRehabilitation 2005;20: 205-221.

40. Fox CM, Farley BG, Ramig LA, McFarland D. An integrated rehabilitation approach to Parkinson's disease: learning big and loud. Movement Disorders 2005;20:S149.
41. Aparicio P, Diedrichsen J, Ivry RB. Effects of focal basal ganglia lesions on timing and force control. Brain Cogn 2005;58:62-74.

42. Iansek R, Huxham F, McGinley J. The sequence effect and gait festination in Parkinson disease: contributors to freezing of gait? Mov Disord 2006;21:1419-1424.

43. Mawdsley C, Gamsu CV. Periodicity of speech in Parkinsonism. Nature 1971;231:315-316.

44. De Letter M, Vantens P, Van Borsel J. The effects of levodopa on word intelligibility in Parkinson's disease. J Commun Disord 2005;38:187-196.

45. Hughes AJ, Daniel SE, Kilford L, Lees AJ. Accuracy of clinical diagnosis of idiopathic Parkinson's disease: a clinico-pathological study of 100 cases. J Neurol Neurosurg Psychiatry 1992;55:181184.

46. Kokmen E, Naessens JM, Offord KP. A short test of mental status--description and preliminary results. Mayo Clin Proc 1987; 62:281-288.

47. Fahn S, Elton RL, Committee Ud. Unified Parkinson's Disease Rating Scale. In: Fahn S, Marsden CD, Calne DB, Lieberman A, editors. Recent developments in Parkinson's disease. Florham Park, NJ: Macmillan; 1987. p 153-163.

48. Langston JW, Widner H, Goetz CG, et al. Core assessment program for intracerebral transplantations (CAPIT). Mov Disord 1992;7:2-13.

49. Carson CP, Ingrisano DR, Eggleston KD. The effect of noise on computer-aided measures of voice: a comparison of CSpeechSP and the multi-dimensional voice program software using the CSL 4300B module and multi-speech for windows. J Voice 2003;17: 12-20.

50. Butterworth B. Evidence from pauses in speech. In: Butterworth B, editor. Language production. London: Academic Press; 1980. p 155-176.

51. Larson K, Ramig L, Scherer R. Acoustic and glottographic voice analysis during drug-related fluctuations in Parkinson's disease. J Med Speech Lang Pathol 1994;2:227-239.

52. Daniels N, Oates J, Phyland DJ, Feiglin A, Hughes A. Vocal characteristics and response to levodopa in Parkinson's disease. Mov Disord 1996;11 (Suppl 1):117.

53. Kompoliti K, Wang QE, Goetz CG, Leurgans S, Raman R. Effects of central dopaminergic stimulation by apomorphine on speech in Parkinson's disease. Neurology 2000;54:458-462.

54. Viallet F, Teston B, Ludovic J, et al. Effects of pharmacological versus electrophysiological treatments on Parkinsonian dysprosody. In Speech Prosody, April 11-13, Aix-en-Provence, 2002.

55. Jiang J, Lin E, Wang J, Hanson DG. Glottographic measures before and after levodopa treatment in Parkinson's disease. Laryngoscope 1999;109:1287-1294.

56. Schulz GM. The effects of speech therapy and pharmacological treatments on voice and speech in Parkinson s disease: a review of the literature. Curr Med Chem 2002;9:1359-1366.

57. Goberman A, Coelho C, Robb M. Phonatory characteristics of parkinsonian speech before and after morning medication: the ON and OFF states. J Commun Disord 2002;35:217-239.

58. Colosimo C, Merello M, Hughes A, Sieradzan K, Lees A. Motor response to acute dopaminergic challenge with apomorphine and levodopa in Parkinson's disease: implications for the pathogenesis of the on-off phenomenon. J Neurol Neurosurg Psychiatry 1996; 60:634-637.

59. McColl CD, Reardon KA, Shiff M, Kempster PA. Motor response to levodopa and the evolution of motor fluctuations in the first decade of treatment of Parkinson's disease. Mov Disord 2002;17: 1227-1234.

60. Fox CM, Ramig LO. Vocal sound pressure level and self-perception of speech and voice in men and women with idiopathic Parkinson disease. Am J Speech Lang Pathol 1997;6:85-94. 